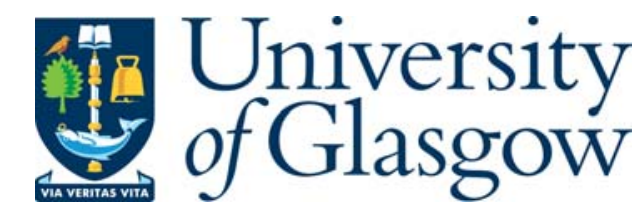

Tan, K.C. and Li, Y. (2002) Grey-box model identification via evolutionary computing. Control Engineering Practice 10(7):pp. 673684.

http://eprints.gla.ac.uk/3808/

Deposited on: 12 November 2007 


\title{
GREY-BOX MODEL IDENTIFICATION VIA EVOLUTIONARY COMPUTING
}

\author{
K. C. Tan \\ Department of Electrical and Computer Engineering \\ National University of Singapore \\ 10 Kent Ridge Crescent, Singapore 119260. \\ E-mail: eletankc@nus.edu.sg \\ Y. Li \\ Centre for Systems and Control, and \\ Department of Electronics \& Electrical Engineering \\ University of Glasgow, Glasgow G12 8LT, UK. \\ E-mail: y.li@elec.gla.ac.uk
}

\begin{abstract}
This paper presents an evolutionary grey-box model identification methodology that makes the best use of a-priori knowledge on a clear-box model with a global structural representation of the physical system under study, whilst incorporating accurate black-box models for immeasurable and local nonlinearities of a practical system. The evolutionary technique is applied to building dominant structural identification with local parametric tuning without the need of a differentiable performance index in the presence of noisy data. It is shown that the evolutionary technique provides an excellent fitting performance and is capable of accommodating multiple objectives such as to examine the relationships between model complexity and fitting accuracy during the model building process. Validation results show that the proposed method offers robust, uncluttered and accurate models for two practical systems. It is expected that this type of grey-box models will accommodate many practical engineering systems for a better modelling accuracy.
\end{abstract}

\section{KEYWORDS}

System identification, grey-box models, evolutionary algorithms, multi-objective 


\section{INTRODUCTION}

The increasing demands on product 'quality' and 'performance' nowadays have generated a greater emphasis of knowledge acquisition for engineering systems. In many instances, this knowledge is modelled by a set of linear ordinary differential equations (ODEs) derived from the physical principles or operating mechanism of the system (Kemna and Mellichamp, 1995; Mattsson et al., 1998; Vandemolengraft et al., 1994). Modelling based on the ODEs or the clear-box model as referred in this paper has the advantage of representing a wide range of nonlinear systems whilst retaining useful system structural information. Practical systems are, however, usually complicated and may involve unknown noises, nonlinearities and unseen dynamics that often appear as lumped modelling errors (Funkquist, 1997; Gawthrop et al., 1993; Tan et al., 1997). It is thus often impossible to identify every detailed nonlinearity to build an accurate clear-box model for a dynamic system.

In contrast to the clear-box, black-box models such as nonlinear auto-regressive moving average or artificial neural network models may be employed to approximate a nonlinear system (Chen et al., 1990; Leontaritis and Billings, 1985). However, physical significance or structural information of the system will be lost if such black-box models are attempted (Gawthrop et al., 1993; Gray et al., 1998), since mappings between a black-box model and the set of ODEs of a nonlinear system are not bijective or equivalent. This unclear system representation highly restricts the system analysis to be carried out and is thus less applicable for control purposes.

The underlying nonlinearities of a practical engineering system and some of its physical parameters are usually known a-priori. However, some of the, mostly minor, nonlinearities cannot be modelled accurately due to the system complexity and constraints on physical ability to measure. This is thus seen as a partially known system and may be best modelled as a grey-box (Forsell and Lindskog, 1997; Gawthrop et al., 1993; Sjöberg and Raedt, 1997; Li et al., 1997). Since obtaining accurate system ODEs that have a focused nonlinear structure is desired in many applications (Gray et al., 1998; Kemna and Mellichamp, 1995), a grey-box model to be established should explicitly utilise the a-priori knowledge such as those based on the clear nonlinear structure and parameters derived from physical laws. This forms the 'clear' part of the grey-box and the 'black' part will be used to approximate any neglected or immeasurable nonlinearities in the system. In such a grey-box representation, system structure will not be replaced by artificial structures as seen in the generic 
black-box approximators. Hence, this modelling technique is different from the conventional clear or black-box models and should offer advantages over those methods.

Such grey-box model identification is often a multi-modal optimisation problem in a multidimensional space. The optimisation task is not suitable for conventional gradient-guided techniques, which require a differentiable objective function in a smooth search space. In addition, these techniques may encounter difficulties due to noisy data or system discontinuity, and may only offer "local optima" if the initial guess is inappropriate (Fonseca, 1995; Gray et al., 1996; Kristinsson and Dumont, 1992; Maclay and Dorey, 1993; Tan et al., 1997; Yao and Sethares, 1994). These numerical difficulties can, however, be easily overcome by Darwinian-Wallace principle based evolutionary optimisation that explores a poorly understood solution space in parallel by intelligent trials without the need of differentiating the performance index or linearly separable parameters (Fogel, 1995; Michalewicz, 1994). Moreover, the evolutionary algorithm is capable of incorporating Pareto's optimality for multi-objective optimisation that allows engineers to examine different trade-offs between model complexity and fitting accuracy before final determination of a suitable grey-box model structure with optimised parameters optimised according to the engineer's preferences.

A simple and efficient grey-box model identification technique is developed in this paper, which is tractably enabled by genetic evolution and local learning. The evolutionary grey-box model identification is further extended for multi-objective optimisation to study the relationships between model complexity and fitting accuracy. The methodology is detailed with two nonlinear model identification examples, including a hydraulic nonlinear system in Section 2 and a neutron intensity control system in Section 3. Conclusions are drawn in Section 4.

\section{A COUPLED NONLINEAR SYSTEM IDENTIFICATION PROBLEM}

\subsection{The System and Measured Clear-Box Model}

A nonlinear coupled twin-tank hydraulic system that may also be extended to simulate heat-balance in chemical processes is shown in Fig. 1. Based on the physical law of Bernoulli's mass-balance equation, a clear-box model of the system is given by (Tan, 1997) 


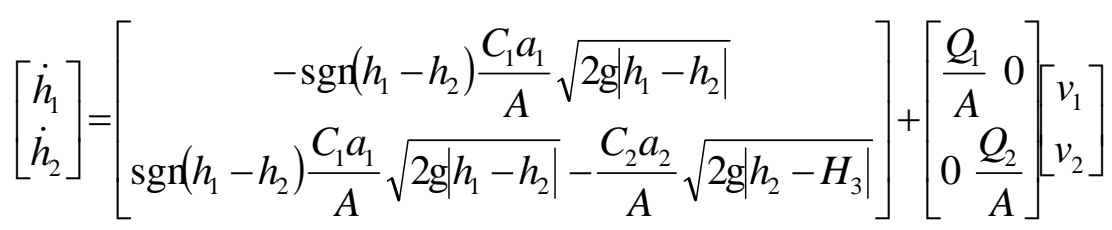

Here the tanks are linked through a coupling pipe of an equivalent orifice area $a_{1}$; the equivalent discharging area of Tank 2 is modelled by $a_{2}$; the liquid level in Tank 1 is $h_{1}$; that in Tank 2 is $h_{2}$ with a physical constraint being $h_{2}>H_{3}$, the equivalent height of both the coupling and discharging pipes; $C_{1}$ and $C_{2}$ are equivalent discharge constants; $A=100 \mathrm{~cm}^{2}$ is the cross-sectional area of both tanks (which can be physically measured with a relatively high accuracy); $Q_{1}$ and $Q_{2}$ are the input flow rate per actuating volt of the pre- and power amplifiers for Tank 1 and Tank 2, respectively; and $\mathrm{g}=981 \mathrm{~cm} \mathrm{~s}^{-2}$ the gravitational constant. Based on the manufacturer's specification and further physical measurements at an operating level of $\left[v_{1}, v_{2}\right]=[2.5 \mathrm{~V}, 2.5 \mathrm{~V}]$, the measured model parameters are shown in the second column of Table 1.

[Table 1 here]

[Fig. 1 here]

To study this clear-box model obtained by the physical law and actual measurements, steps plus delayed small pseudo random binary sequences (PRBS) (Tan et al., 1997) were first used to excite the physical system at the operating levels of $\left[v_{1}, v_{2}\right]=[2.5 \mathrm{~V}, 2.5 \mathrm{~V}]$ and $\left[v_{1}, v_{2}\right]=[2.5 \mathrm{~V}, 1.5 \mathrm{~V}]$, respectively. The PRBS inputs were used in supplement to the steps to excite the high frequencies, and techniques for selecting the PRBS for the relevant frequency range were recommended by Tan (1997). The water levels of the two physical tanks, $h_{1}$ and $h_{2}$, were measured and shown in Fig. 2.

[Fig. 2 here]

The same input pattern of steps plus PRBS at the operating level of $\left[v_{1}, v_{2}\right]=[2.5 \mathrm{~V}, 2.5 \mathrm{~V}]$ are then applied to the measured clear-box model. The absolute error residuals between the actual and measured model responses due to the model inaccuracy, $e_{1}$ and $e_{2}$, are shown in Fig. 3(a). To study the measured model accuracy and robustness at different operating level, the absolute error residuals between the actual and model responses at the operating point of $\left[v_{1}, v_{2}\right]=[2.5 \mathrm{~V}, 1.5 \mathrm{~V}]$ 
are shown in Fig. 3(b). The corresponding root mean-square (RMS) errors, i.e., $R M S=\sqrt{\frac{\sum_{i=1}^{n} e_{i}}{n}}$, where $n$ is the number of data points and $e$ is the error between the actual and model responses, are given in the third row of Table 2. Although the measured clear-box is a nonlinear model, it can be seen that the errors are large and much dependent on the operating points. Moreover, due to the unknown and unattainable nonlinearities, empirical measuring shows that the ODE coefficients, $C_{1}$ and $C_{2}$, are also operating-point dependent as indicated in Fig. 4.

\section{[Table 2]}

[Fig. 3 here]

[Fig. 4 here]

\subsection{Identification of the Clear-Box Model}

\subsubsection{Quasi-Newton's search method}

To derive an accurate clear-box model, the well-known gradient-guided search method of BFGS (Broydom, 1970; Fletcher, 1970; Golfarb, 1970; Shanno, 1970) Quasi-Newton's algorithm in Matlab optimisation toolbox (Grace, 1999) is used to identify the clear-box model in (1). Similar inputs of steps plus PRBS at the operating level of $\left[v_{1}, v_{2}\right]=[2.5 \mathrm{~V}, 2.5 \mathrm{~V}]$ were used to identify the clear-box model of (1) using the Quasi-Newton's method such that the modelling error:

$$
J=\left\|W_{1}(t) e_{1}(t)\right\|+\left\|W_{2}(t) e_{2}(t)\right\|
$$

is minimised. $W_{1}$ and $W_{2}$ can be distinctive weighting functions, which are here chosen to be 1 since no emphasis on transient or steady-state is placed. Without loss of generality, the $L_{2}$ norm (similar to the RMS value) is used in this work, as linear norms in the Euclidean space are mutually bounded (i.e., metric equivalence).

The Quasi-Newton's method has however, converged to local optima and failed to identify an optimal clear-box model due to the nonlinear and sensory noisy data of the system. With different trial-and-error initial conditions, the best recommended "local" parameters from the QuasiNewton's method are shown in the third column of Table 1. The absolute error residuals between the actual and model responses are shown in Fig. 5(a). To validate the model at different operating 
point, error residuals at the operating level of $\left[v_{1}, v_{2}\right]=[2.5 \mathrm{~V}, 2.5 \mathrm{~V}]$ are plotted in Fig. 5(b). The best local convergence trace of the Quasi-Newton's method is shown in Fig. 6, and the corresponding RMS errors at the two different set of operating levels are shown in the fourth row of Table 2. It can be seen that the Quasi-Newton's method has failed in this identification task with a much larger RMS errors than the measured model obtained from the manufacturer's specifications.

[Fig. 5 here]

[Fig. 6 here]

\subsubsection{Evolutionary optimisation approach}

Evolutionary algorithm (EA) based identification technique has been applied to identify the clearbox model of (1). EA evaluates performances of candidate solutions at multiple points simultaneously and thus efficiently approaches the global optimum. Before this simulated evolution process begins, an initial population of multiple chromosomes representing random candidate models is formed. Every such chromosome is assigned a fitness function and at each generation of search, multiple candidates are evaluated and the search will be directed intelligently according to the Darwin's “survival-of-the-fittest” principle. Then useful search information and co-ordinates are exchanged and altered for the next generation of candidate solutions. This evolution cycle will be repeated until the final generation is reached or the solution has been found. For details of EA, readers may refer to Michalewicz (1994).

Since gradient information can be estimated from the function evaluation (Adby and Dempster, 1974), the mutation operation in EAs can be further fine-tuned or realised by means of the QuasiNewton's algorithm (Grace, 1999) or Boltzman's annealing criteria (Li et al., 1997) to overcome the problems of chromosome stagnation and weak local exploration of a standard EA. Here, decimalcoding scheme is used to reduce the chromosome length and to avoid the Hamming-cliff effect often encountered in a binary-coding based EA (Li et al., 1997). The multiple-decoding scheme (Tan, 1997) with adaptive selection of searching range has been adopted in the paper, e.g., each parameter is encoded by 4 digits, 3 of which represent the number of quantified values of the parameters within a given range selection. The extra digit is used as a "control gene" to search for an appropriate range of that parameter, which benefits the EAs to have finer resolution and easy setting of the parameter range. The mutation is applied at a probability rate of 0.1 and due to the increased local fine-tuning activity in the hybrid EA (Li et al., 1997), the crossover rate is reduced and set as 
0.5 in a similar way to evolution strategy techniques (Schwefel, 1995). Here a randomly positioned standard single-point crossover and tournament selection scheme (Li et al., 1997) are employed for rapid reproduction, where two random chromosomes compete once for survival.

The same objective function of (2) was adopted in the evolutionary identification, and it took about 2 hours for the hybrid EA to run for 100 generations with a population size of 100 on a $200 \mathrm{MHz}$ Pentium Pro processor. The obtained clear-box model parameters are shown in the fourth column of Table 1. Fig. 7 shows the convergence trace of the best $10 \%$ of the identified parameters at each generation. The candidate values of a parameter within the best $10 \%$ models converged to a narrow range. It can be observed from Fig. 7 that the hybrid evolution managed to escape from a distinctive local optimum mainly caused by $C_{2} a_{2}$ and $Q_{2}$ after 20 generations, which shows the effectiveness of the evolutionary based identification techniques.

[Fig. 7 here]

The resulting error residuals for the evolutionary identification are shown in Fig. 8, and their corresponding RMS errors at the two set of different operating levels are given in the fifth row of Table 2. Since the data used for the identification are at operating level of $\left[v_{1}, v_{2}\right]=[2.5 \mathrm{~V}, 2.5 \mathrm{~V}]$, the EA identified clear-box model offers a better fitting accuracy than the measured and gradientoptimised model at this operating point (c.f. Figs. 3(a), 5(a) and 8(a)). However, the validation also indicates that the EA identified clear-box model had failed to offer a similar accuracy if the operating point tested is different from that used in obtaining the model (c.f. Figs. 3(b), 5(b) and 8(b)). Although the clear-box is a nonlinear model, validation shows that it is not suitable in representing the coupled twin-tank system, as it ignores the immeasurable non-dominant nonlinearities involved in the system.

[Fig. 8 here]

\subsection{A Novel Type of Grey-Box Model}

The nonlinearities that have often been omitted in such a model identification exercise include nondominant contributions by fluid tension or friction, as well as simplification from distributed pipes to lumped orifices. Other inaccuracies may arise from manufacturing tolerance and measurement errors. The dependency of the ODE coefficients on operating points is, in fact, a state dependency 
determined by the potential energies of $2 \mathrm{~g}\left|h_{1}-h_{2}\right|$ and $2 \mathrm{~g}\left|h_{2}-H_{3}\right|$. As shown in Fig. 4, the two discharge coefficients of $C_{1}$ and $C_{2}$ at the steady-state are nonlinearly dependent on the potential energies of $2 \mathrm{~g}\left|h_{1}-h_{2}\right|$ and $2 \mathrm{~g}\left|h_{2}-H_{3}\right|$, respectively.

For engineering applications such as the coupled liquid-level system, it is often impossible to identify every detailed nonlinearity to obtain an accurate clear-box model. However, it is often desired to have a focused physical model structure of the system (Vandemolengraft et al., 1994). For this, a grey-box model which builds from a clear-box as the global structure and incorporates local black-boxes to model the operating-point sensitive coefficients that are unable to be modelled by a clear-box due to the neglected nonlinearities is proposed here. Unlike existing grey-box structure, such black-boxes are coupled with sensitive or immeasurable clear-box parameters and can be in the form of a power series polynomial, fuzzy logic, neural network or other types of basis function based generic function approximators. Since Padé approximation in the form of a regressive function is accurate and efficient, it is recommended here to play as the black-box role in the combined grey-box model.

For (1), the two sensitive “coefficients” are now modelled by the black-box as

$$
\frac{C_{1} a_{1}}{A}=\frac{C_{10}+c_{11}\left|h_{1}-h_{2}\right|+c_{12}\left|h_{1}-h_{2}\right|^{2}+\cdots}{A\left(1+d_{11}\left|h_{1}-h_{2}\right|+d_{12}\left|h_{1}-h_{2}\right|^{2}+\cdots\right)}
$$

and

$$
\frac{C_{2} a_{2}}{A}=\frac{c_{20}+c_{21}\left|h_{2}-H_{3}\right|+c_{22}\left|h_{2}-H_{3}\right|^{2}+\cdots}{A\left(1+d_{21}\left|h_{2}-H_{3}\right|+d_{22}\left|h_{2}-H_{3}\right|^{2}+\cdots\right)}
$$

where $c_{i j}$ and $d_{i j}$ are the coefficients to be determined in the grey-box identification.

\subsection{Identification of the Grey-Box Model}

For the resultant grey-box model that combines (1), (3) and (4), the identification task is to find the coefficients $H_{3}, Q_{1}, Q_{2}, c_{i j}$ and $d_{i j}$ such that the objective function of (2) is minimised. For simplicity, the Padé polynomial in (3) and (4) is limited to be second-order polynomial. Both Quasi-Newton's method and hybrid EA have been applied to identify the grey-box model at an 
operating point $\left[v_{1}, v_{2}\right]=[2.5 \mathrm{~V}, 2.5 \mathrm{~V}]$, and their respective recommended parameters are shown in Table 3. Since $H_{3}$ in (1) cannot be linearly parameterised or separated from $C_{2} a_{2}$ and the blackboxes need to be identified simultaneously within the nonlinear clear-box model structure, the Quasi-Newton's method has failed to search for an optimal grey-box model. The resulting error residuals for the Quasi-Newton's method are large as indicated in Fig. 9, and the corresponding RMS errors at the two set of different operating points are given in the sixth row of Table 2. Fig. 10 shows the best local convergence trace of the Quasi-Newton's method.

The resulting error residuals for the hybrid EA identified grey-box model are shown in Fig. 11, and its RMS values at different operating point are listed in the seventh row of Table 2. It can be seen that the hybrid EA identified grey-box model not only provides significantly improved identification quality but also robust to different operating levels. More importantly, the grey-box had also revealed the nonlinear trends of $C_{1} a_{1}$ and $C_{2} a_{2}$ as the potential energies changes as shown in Fig. 12 (c.f. Figs. 4 and 12). In the evolutionary identification process, candidate models in the evolution can combine physical and empirical models (Kemna and Mellichamp, 1995), and the evolution can start from empirical ones even if they are only clear-box models initially.

[Table 3 here]

[Fig. 9 here]

[Fig. 10 here]

[Fig. 11 here]

[Fig. 12 here]

\section{A MEASUREMENT SYSTEM MODELLING PROBLEM}

\subsection{The Neutron Intensity Control System}

A reflectivity system based on collimated radiation is used in Bioengineering to determine the thickness and structural characteristics of thin films. A neutron beam hitting the film sample is reflected to the monitoring detector as shown in Fig. 13. To get a complete description of the reflectivity profile, the sample is rotated so that the incident beam strikes it at different angles. 
Because the rotation will vary the intensity of the reflected beam, it is necessary to control the size of the neutron beam hitting the reflecting sample by means of two slits $S_{1}$ and $S_{2}$ while the sample is rotated. To analyse the thin film sample using its reflectivity, the intensity of the beam hitting the sample, $A_{d}$, must be known so that calibrations can be made. This is difficult for arbitrary combination sizes of $S_{1}$ and $S_{2}$ values. Therefore an inverse model needs to be generalised for automatic calibration of the reflectivity system.

[Fig. 13 here]

\subsection{Clear-Box Model Representation and Identification}

To generalise the calibration model for arbitrary combination sizes of $S_{1}$ and $S_{2}$, the intensity responses to more than 60 pre-determined combination sizes of $S_{1}$ and $S_{2}$ are obtained as training data. To measure the data accurately within the sensitivity range of the detector while the slit sizes are changed, the manufacturer of the system recommends adding an attenuator after the Slit 2. However, the attenuation is suggested to be varied with the product of the two slit sizes by 5 discretely decreasing values as shown in Table 4.

[Table 4 here]

By physical principles, the 'gain', $I$, from the input beam intensity, $A_{s}$, to the output beam intensity, $A_{d}$, is proportional to the product of the cascaded slit sizes. A clear-box model for the neutron intensity system may thus be established as,

$$
I=\frac{A_{d}}{A_{s}}=c \cdot k_{i} \cdot S_{1} \cdot S_{2}
$$

where $c=7.63$ is a proportional factor of the gain depending upon the source intensity and the detector sensitivity. It is used to model any neglected factors that may not be measured accurately, such as the diffraction in a lumped manner; $k_{i}$ denotes the discretely decreasing attenuating factor as given in Table 4; $S_{1}$ and $S_{2}$ is the size of the cascaded slits 1 and 2, respectively.

To identify the clear-box model in (5), the physical neutron intensity experiment was repeated three times to collect three sets of experimental I/O data with different combination sizes of slits $S_{1}$ and 
$S_{2}$, as to average down the measurement errors. The task of the identification is thus to obtain an optimal set of $k_{i}$ such that the following quadratic objective function

$$
J=\sum_{m=1}^{3} \sum_{n}\left(I_{n}-\hat{I}_{n}\right)^{2}
$$

is minimised. Where $\hat{I}$ represents the measured output data; $m$ denotes the repeated number of experiments; $n>60$ denotes the number of measurements made in one data set.

Both the gradient-guided Quasi-Newton's method and evolutionary computing approach have been applied to identify the clear-box model in (5). The estimated attenuating factor $k_{i}$ is given in Table 5. It can be seen that the two methods provide an equally good model with similar coefficient values, which is likely to be the global optimum. However, their corresponding RMS errors as shown in Table 5 are rather large $(\approx 150)$. This is also reflected by the poor fitting accuracy between the data index of 40 and 60 as shown in Fig. 14, which suggests that the clear-box model of (5) cannot accurately represent the neutron intensity system.

[Table 5 here]

[Fig. 14 here]

\subsection{Grey-Box Model Representation and Identification}

The neglected diffraction effects and other unknown factors in the clear-box model may, however, be accounted by using a Padé approximation based black-box model as discussed in Section 2. Based on this, an accurate grey-box model may be established in order to include diffraction and other neglected and difficult-to-model effects. The clear-box structure of (5) is preserved and a general black-box structure is included in the formation of the grey-box model as given by

$$
I=c \cdot k_{i} \cdot \frac{\left(a_{0}+a_{1} S_{1}+a_{2} S_{2}\right)\left(b_{0}+b_{1} S_{1}+b_{2} S_{2}\right)}{c_{0}+c_{1} S_{1}+c_{2} S_{2}}
$$

where $a_{i}, b_{i}$, and $c_{i}$ are the model coefficients to be determined. Note that the inclusion of the denominator may be unnecessary but could be useful for including some insignificant general nonlinearities. The same data set of $\hat{I}$ in Section 3.2 is used here to determine the grey-box 
coefficients of the neutron intensity system, and the aim is to find the best set of $a_{i}, b_{i}, c_{i}, k_{i}$ in (7) such that the amalgamated quadratic identification errors in (6) are minimal.

Both the Quasi-Newton and evolutionary computing methods were used to identify an optimal grey-box model of (7). The resulting grey-box model coefficients and RMS errors are shown in Table 5. Again, both methods resulted in identical RMS errors, which is likely to be the global optimum. As shown in Table 5, the grey-box identification has multiple solutions or peaks as discovered by the hybrid EA. Since EA explores multiple points in the solution space in parallel, it has the unique capability of identifying multiple equal or unequal peaks, which is unmatched by conventional gradient-guided techniques (Michalewicz, 1994). The output response of the grey-box model with coefficient set of peak 1 in Table 5 is shown in Fig. 15. Obviously, the identified greybox model has performed a very good fitting to the neutron intensity system. Moreover, the greybox identification has produced an RMS error of 77.6, which is much smaller than the RMS error of 150 in the clear-box model due to its neglected non-dominant characteristics. Note that the results also correctly yielded a relatively small value of $a_{0} \cdot b_{0}$, as $S_{1}=S_{2}=0$ would result in a zero brightness count.

\section{[Fig. 15 here]}

\subsection{Trade-Offs Among the Trajectory}

In Fig. 15, there are 5 different trajectories resulted from the different switching of attenuating factor $k_{i}$. By providing a compromised model for the entire trajectory with the smallest amalgamated errors in (6), a grey-box model of (7) may be obtained. However, one coefficient set of $a_{i}, b_{i}, c_{i}, k_{i}$ in (7) that is accurately fitted to one of the trajectory may not be the same for the others. To examine the relationships among the different trajectory, multi-objective optimisation is necessary such that a set of trade-off models could be obtained and visualised before final determination of a model based upon the on-hand situation. Unlike conventional gradient-guided optimisation techniques, evolutionary algorithm can be easily extended for multi-objective optimisation in view of its parallel-based evolution feature (Fonseca, 1995; Tan et al., 1999). A multi-objective evolutionary algorithm is capable to approximate the set of non-dominated solutions known as Pareto optimal set, where each objective component of any model along the Pareto front can only be improved by degrading at least one of its other objective components. 
Here, fitting errors for each of the trajectory is regarded as one of the 5 objectives to be minimised in the multi-objective model identification. The MOEA toolbox developed by Tan et al., (2001) has been used here to find the set of Pareto optimal solutions, which is freely available for download at http://vlab.ee.nus.edu.sg/ kctan/moea.htm. The toolbox implements the multi-objective evolutionary algorithm (MOEA) proposed by Tan et al., (1999) with goal-sequence domination scheme that allows the incorporation of advanced hard/soft priority and constraint information on each of the objective components. Besides, the MOEA includes a dynamic sharing scheme that is simple and adaptively estimated according to the on-line population distribution without the need of any a-priori parameter setting. The toolbox is fully functional with graphical user interface (GUI) and is ready for immediate use without much knowledge on evolutionary computing. It also allows the generation of simulation results in various formats, such as text files or graphical displays for the purpose of on-line viewing and analysis. In addition, a file handling capability for saving all simulation results and model files in a Mat-file format for Matlab or text-file format for software packages like Microsoft Excel is also available.

It took less than 0.5 hour for the evolution to run for 100 generations with a population size of 50 on a $1 \mathrm{GHz}$ Pentium III processor. All individuals obtained at the end of the evolution are consists of non-dominated models, indicating a substantial trade-offs among the 5 objectives. The trade-off graph of these non-dominated models is shown in Fig. 16, where each line representing a solution found by the MOEA. In the figure, trade-offs between adjacent objective results in the crossing of lines between them; whereas concurrent lines indicate that the objectives do not compete with each other. As can be seen, the lines are crossing between any two adjacent objectives showing the tradeoffs of fitting accuracy among any of the 5 trajectory. The capability of MOEA in finding the set of non-dominated models has thus provided a greater flexibility for control engineers in determining the most preferable model with the best fitting accuracy for a particular trajectory of the neutron intensity system depending on his/her preference or job-on-hand.

\section{[Fig. 16 here]}

\subsection{Determination of Model Complexity}

Alternatively, the grey-box model structure of (7) may be represented by other Padé approximation or expanded to a more general higher order polynomial. Although complex model structure with higher orders may result in better fitting accuracy than a lower order model, sophisticated model 
structure is often undesirable in system identification (Ljung, 1987; Söderström and Stoica, 1989). To determine an appropriate grey-box model structure with optimal model coefficients, the MOEA toolbox (Tan et al., 2001) was applied here to study the relationships of model complexity and fitting accuracy. For this, the grey-box model structure of (7) was expanded systematically from first-order to seventh-order as listed in Table 6. Note that the clear-box model of $\mathrm{MC}_{3}$ was also included in the expansion list to provide an easy comparison with other model complexities.

The objective function of (6) for each of the grey-box model structure in Table 6 is regarded as an independent objective for the optimisation, which results in 7 objectives to be optimised concurrently in the evolution. To encourage the MOEA to consistently evolve optimal model parameters for all model structures, the best models for each of the 7 model structures at each generation were preserved and evolved into the next generation without any genetic operations, i.e., an elitism strategy was used in the evolution (Tan et al., 1999). Here, a model complexity index ranging from 1 to 7 was included for turning on/off any unnecessary model coefficients as appropriate. The algorithm has been run for 100 generations with a population size of 100, and the best models for each of the model structures at the end of the evolution are shown in Table 6 .

The graph of RMS errors versus the model complexity index is shown in Fig. 17. It can be seen that the RMS errors are decreased with the increased of model complexity index, which indicates the trade-offs between model complexity and fitting accuracy for the neutron intensity system. Further, it is shown that the RMS errors are decreased and saturated at the model complexity level of $\mathrm{MC}_{6}$. This suggests that further expansion of the polynomial beyond the structure of $\mathrm{MC}_{6}$ is unnecessary, and thus the grey-box model with structure $\mathrm{MC}_{6}$ may be regarded as a good representation for the neutron intensity system. It should be noted that the exact global optimum is unknown for the neutron intensity modelling problem based upon the practical real data. To verify the optimality of the nondominated front in Fig. 17, individual simulations for the different model complexity using the Quasi-Netwon's method with different initial conditions have been performed. Results show that the individual optimum for each of the model complexity using the Quasi-Netwon's method is very similar (within 1\% variance) to the nondominated front as shown in Fig. 17.

[Table 6 here]

[Fig. 17 here] 


\section{CONCLUSIONS}

A novel cost-effective evolutionary grey-box model identification technique has been presented in the paper. Such a grey-box model directly utilises the physical law based clear-box dominated global structure, with local black-boxes to include immeasurable and local nonlinearities of a practical system. The method offers an accurate, uncluttered and robust model with insightful representation of nonlinearities, which cannot be matched by conventional means.

It has been shown that the grey-box model identification may be failed by conventional gradientguided model fitting methods, but can be easily established through global optimisation techniques by evolutionary computing. The evolution can start from empirical models, making the best use of existing knowledge on a practical system. In addition, the evolutionary technique is capable of accommodating multiple objectives to examine different trade-offs between the model complexity and fitting accuracy before final determination of a suitable grey-box model structure with optimised parameters.

Two practical applications of a hydraulic nonlinear system and a neutron intensity control system have shown good feasibility and accuracy of the grey-box models. It is expected that the proposed multi-objective evolutionary grey-box identification technique could be easily applied to obtain accurate nonlinear models and better representations for many industrial plants.

\section{ACKNOWLEDGMENT}

The authors wish to thank G. W. Irwin and the anonymous reviewers for their valuable comments and helpful suggestions which greatly improved the paper quality.

\section{REFERENCES}

Adby P. R. and Dempster M. A. H. (1974). Introduction to optimization methods, Chapman and Hall, London. 
Broydom C. G. (1970). The Convergence of a class of double-rank minimization algorithms, J. Inst. Math. Applic., 6, 76-90.

Chen, S., Billings, S. A. and Grant, P. M. (1990). Non-linear system identification using neural networks, Int. J. Control, 51, 1191-1214.

Fletcher R. (1970). A new approach to variable metric algorithms, Computer Journal, 13, 317-322.

Fogel, D. B. (1995). Evolutionary Computation, IEEE Press, Piscataway, NJ.

Fonseca C. M.. (1995). Multiobjective Genetic Algorithms with Application to Control Engineering Problems, Ph.D. Thesis, Dept. Automatic Control and Systems Eng., Univ. of Sheffield, UK.

Forsell, U. and Lindskog, P. (1997). Combining semi-physical and neural network modelling: An example of its usefulness, In Preprint, $11^{\text {th }}$ IFAC Symposium on System Identification, Kitakyushu, Japan, 4, 795-798.

Funkquist, G. (1997). Grey-box identification of a continuous digester - A distributed parameter process, Contr. Eng. Pract., 5, (7), 919-930.

Gawthrop, P. J., Jezek, J., Jones, R. W. and Sroka, I. (1993). Grey-box model identification, Invited Paper, Control-Theory and Advanced Tech., 9, (1), 139-157.

Golfarb D. (1970). A family of variable metric updates derived by variational means, Mathematics of Computing, 24, 23-26.

Grace, A. (1999). Optimisation Toolbox for Use with MATLAB, Version 2, The MathWorks Inc.

Gray, G. J., Murray-Smith, D. J., Li, Y. and Sharman, K. C. (1996). Structural system identification using genetic programming and a block diagram oriented simulation tool, Electronics Letters, 32, (15), 1422-1424.

Gray, G. J., Murray-Smith, D. J., Li, Y., Sharman, K. C. and Weinbrenner T. (1998). Identification of a Grey box model of nonlinear current transformers for simulation purposes, Contr. Eng. Pract., 6, (11).

Kemna, A. H. and Mellichamp, D. A. (1995). Identification of combined physical and empiricalmodels using nonlinear a-priori knowledge, Contr. Eng. Pract., 3, (3), 375-382.

Kristinsson, K. and Dumont, G. A. (1992). System identification and control using genetic algorithms, IEEE Trans. Syst. Man and Cyber., 22, (5), 597-602.

Leontaritis, I. J. and Billings, S. A. (1985). Input-output parametric models for non-linear systems. Part I: deterministic non-linear systems, Int. J. Control, 41, 303-328.

Li, Y., Tan, K. C. and Gong, M. R. (1997). Global structure evolution and local parameter learning for control system model reductions, Evolutionary Algorithms in Eng. Appl., Dasgupta, D. and Michalewicz, Z. (Eds.), Springer Verlag, 345-360. 
Ljung, L. (1987). System Identification: Theory for the User, Prentice-Hall, Englewood Cliffs, NJ.

Maclay, D. and Dorey, R. (1993). Applying genetic search techniques to drivetrain modeling, IEEE Contr. Syst. Mag., 13, (3), 50-55.

Mattsson, S. E., Elmqvist, H. and Otter, M. (1998). Physical system modelling with Modelica, Contr. Eng. Pract., 6, (4), 501-510.

Michalewicz, Z. (1994). Genetic Algorithms + Data Structures = Evolution Programs, SpringerVerlag, Berlin, $2^{\text {nd }}$ Ed.

Schwefel, Hans-Paul (1995). Evolution and Optimum Seeking, New York, Wiley \& Sons.

Shanno, D. F. (1970). Conditioning of Quasi-Newton methods for function minimization, Mathematics of Computing, 24, 647-656.

Sjöberg, J. and Raedt, R. De. (1997). Nonlinear system identification: a software concept and examples, In Preprint, $11^{\text {th }}$ IFAC Symposium on System Identification, Kitakyushu, Japan, 4, pp. 685-690.

Söderström, T. and Stoica, P. (1989). System Identification, Prentice-Hall International, Hemel Hempstead, Hertfordshire.

Tan, K. C. (1997). Evolutionary methods for modelling and control of linear and nonlinear systems, Ph.D. thesis, Dept. of Electronics \& Electrical Engineering, Univ. of Glasgow, UK.

Tan, K. C., Li, Y., Gawthrop, P. J. and Glidle, A. (1997). Evolutionary grey-box modelling for practical systems, Second IEE Int. Conf. on GA in Eng. Syst. Innovations and Appl., Glasgow, UK, 369-375.

Tan, K.C., Lee, T.H. and Khor, E.F. (1999). Evolutionary algorithms with goal and priority information for multi-objective optimization, Proc. of the IEEE Congress on Evolutionary Computation, 1, Washington, D.C, USA, 106-113.

Tan, K. C., Lee, T. H., Khoo, D. and Khor, E. F. (2001). A multi-objective evolutionary algorithm toolbox for computer-aided multi-objective optimization, IEEE Trans. on Systems, Man and Cybernetics: Part B (Cybernetics), 31, (4), 537-556.

Vandemolengraft, M. J. G., Veldpaus, F. E. and Kok, J. J. (1994). An optimal estimation method for nonlinear mechanical systems, J. Dyn. Syst. Meas. Contr., Trans. ASME, 116, (1), 805-810.

Yao, L. and Sethares, W. A. (1994). Nonlinear parameter-estimation via the genetic algorithm, IEEE Trans. Sig. Proc., 42, (4), 927-935. 
Table 1 The measured and identified clear-box model at operating point $\left[v_{1}, v_{2}\right]=[2.5 \mathrm{~V}, 2.5 \mathrm{~V}]$

\begin{tabular}{|c|c|c|c||}
\hline Clear-Box Model & Measured Values & Quasi-Newton's Method & Hybrid EA \\
\hline$C_{1} a_{1}\left(\mathrm{~cm}^{2}\right)$ & 0.21 & 0.11 & 0.38 \\
\hline$C_{2} a_{2}\left(\mathrm{~cm}^{2}\right)$ & 0.24 & 0.02 & 0.20 \\
\hline$Q_{1}\left(\mathrm{~cm}^{3} \mathrm{~s}^{-1} \mathrm{~V}^{-1}\right)$ & 7 & 0.81 & 7.6 \\
\hline$Q_{2}\left(\mathrm{~cm}^{3} \mathrm{~s}^{-1} \mathrm{~V}^{-1}\right)$ & 7 & 0.79 & 5.1 \\
\hline$H_{3}(\mathrm{~cm})$ & 3 & 0.01 & 2.5 \\
\hline
\end{tabular}


Table 2: The RMS errors of the measured, clear-box and grey-box model

\begin{tabular}{|c|c|c|c|c|c|}
\hline \multicolumn{2}{|c|}{ Mean Excitation Voltages } & \multicolumn{2}{|c|}{$v_{1}=v_{2}=2.5 \mathrm{~V}+\mathrm{PRBS}$} & \multicolumn{2}{|c|}{$\begin{array}{l}v_{1}=2.5 V+\text { PRBS } \\
v_{2}=1.5 V+\text { PRBS }\end{array}$} \\
\hline \multicolumn{2}{|c|}{ RMS Errors (in cm) } & Tank 1: $e_{1}$ & Tank 2: $e_{2}$ & Tank 1: $e_{1}$ & Tank 2: $e_{2}$ \\
\hline \multirow{3}{*}{ Clear-box } & Measured & 1.0470 & 1.5470 & 3.0427 & 1.5117 \\
\hline & Quasi-Newton's method & 5.8429 & 4.7578 & 2.7853 & 1.8369 \\
\hline & Hybrid EA & 0.4613 & 0.5518 & 2.2822 & 2.8844 \\
\hline \multirow{2}{*}{ Grey-box } & Quasi-Newton's method & 1.6492 & 1.1226 & 4.4406 & 6.2240 \\
\hline & Hybrid EA & 0.3402 & 0.3165 & 0.5898 & 0.3154 \\
\hline
\end{tabular}


Table 3: The identified grey-box model parameters

\begin{tabular}{|c|c|c|c|c|}
\hline \multirow{2}{*}{ Padé coefficients } & \multicolumn{2}{|c|}{ Quasi-Newton's method } & \multicolumn{2}{|c|}{ Hybrid EA } \\
\hline & For $C_{1} a_{1}$ & For $C_{2} a_{2}$ & For $C_{1} a_{1}$ & For $C_{2} a_{2}$ \\
\hline$C_{i 0}$ & 0.6759 & -0.1236 & 0.0373 & 0.4004 \\
\hline$c_{i 1}$ & 0.4432 & -0.0657 & 0.3789 & 0.0293 \\
\hline$c_{i 2}$ & 0.8749 & 0.0053 & 0.2773 & 0.0152 \\
\hline$d_{i 1}$ & -0.0131 & -0.1928 & 0.2583 & 0.0811 \\
\hline$d_{i 2}$ & -0.0962 & 0.1012 & 0.8148 & 0.0720 \\
\hline $\mathrm{H}_{3}$ & \multicolumn{2}{|c|}{0.207} & \multicolumn{2}{|c|}{3.1} \\
\hline$Q_{1}$ & \multicolumn{2}{|c|}{0.1977} & \multicolumn{2}{|c|}{7.2} \\
\hline$Q_{2}$ & \multicolumn{2}{|c|}{0.2402} & \multicolumn{2}{|c|}{7.5} \\
\hline
\end{tabular}


Table 4: Discretely decreasing attenuating factors

\begin{tabular}{|c|c|c|c|c|c||}
\hline $\boldsymbol{S}_{\mathbf{1}} \cdot \boldsymbol{S}_{\mathbf{2}}$ & {$[0,0.25)$} & {$[0.25,2.0)$} & {$[2.0,9.0)$} & {$[9.0,44.0)$} & {$[44.0,207.0)$} \\
\hline Attenuating Factors $\boldsymbol{k}_{\boldsymbol{i}}$ & $k_{1}\left(>k_{2}\right)$ & $k_{2}\left(>k_{3}\right)$ & $k_{3}\left(>k_{4}\right)$ & $k_{4}\left(>k_{5}\right)$ & $k_{5}(>0)$ \\
\hline
\end{tabular}


Table 5: The identified model coefficients and RMS errors

\begin{tabular}{|c|c|c|c|c|c|c|c|c|}
\hline \multirow{3}{*}{ Parameters } & \multicolumn{2}{|c|}{ Clear-Box Model } & \multicolumn{6}{|c|}{ Grey-Box Model } \\
\hline & \multirow{2}{*}{ Quasi-Newton } & \multirow{2}{*}{ Hybrid EA } & \multirow{2}{*}{ Quasi-Newton } & \multicolumn{5}{|c|}{ Hybrid EA } \\
\hline & & & & Peak 1 & Peak 2 & Peak 3 & Peak 4 & Peak 5 \\
\hline$k_{1}$ & 3748.1 & 3752.0 & 673.8 & 6473.6 & 4701.9 & 5886.6 & 7037.1 & 696.8 \\
\hline$k_{2}$ & 794.25 & 795.02 & 128.5 & 1234.8 & 896.8 & 1122.8 & 1342.3 & 132.9 \\
\hline$k_{3}$ & 151.42 & 151.64 & 24.9 & 239.5 & 174.0 & 217.8 & 260.4 & 25.8 \\
\hline$k_{4}$ & 28.25 & 28.18 & 5.2 & 50.0 & 36.3 & 45.5 & 54.4 & 5.4 \\
\hline$k_{5}$ & 4.52 & 4.46 & 0.97 & 9.3 & 6.8 & 8.5 & 10.1 & 1.0 \\
\hline$a_{0}$ & & & -4.4 & -0.7 & -0.9 & -0.6 & -1.9 & -4.4 \\
\hline$a_{1}$ & & & 85.5 & 10.3 & 14.3 & 12.2 & 44.9 & 84.9 \\
\hline$a_{2}$ & & & -95.9 & -4 & -8.9 & -15.8 & -66.3 & -95.0 \\
\hline$b_{0}$ & & & -5.46 & -1.7 & -2.0 & -2.0 & -1.0 & -5.3 \\
\hline$b_{1}$ & & & 104.1 & 42.2 & 46.1 & 34.6 & 14.8 & 102.3 \\
\hline$b_{2}$ & & & -114.5 & -63.3 & -65.3 & -30.1 & -7.4 & -114.5 \\
\hline$C_{0}$ & & & 1475.8 & 760.2 & 801.3 & 627.0 & 1232.6 & 1475.3 \\
\hline$c_{1}$ & & & 14.77 & 0.7 & 2.8 & 3.0 & 9.1 & 14.9 \\
\hline$c_{2}$ & & & 96.6 & 70.6 & 68.1 & 50.7 & 90.5 & 96.3 \\
\hline RMS error & 149.5 & 150.1 & & & 77.6 & & & \\
\hline
\end{tabular}


Table 6: Grey-box model coefficients and RMS errors for different model complexity

\begin{tabular}{|c|c|c|c|c|c|c|c|}
\hline $\begin{array}{c}\text { Model } \\
\text { Complexity }\end{array}$ & $\begin{array}{c}\mathrm{MC}_{1}=\frac{n_{1}}{d_{1}}= \\
\frac{a_{0}}{1}\end{array}$ & $\begin{array}{l}\mathrm{MC}_{2}=\frac{n_{2}}{d_{2}}= \\
\frac{n_{1}+a_{1} s_{1}+a_{2} s_{2}}{d_{1}+b_{1} s_{1}+b_{2} s_{2}}\end{array}$ & $\begin{array}{c}\mathrm{MC}_{3}= \\
s_{1} \cdot s_{2}\end{array}$ & $\begin{array}{c}\mathrm{MC}_{4}=\frac{n_{4}}{d_{4}}= \\
\frac{n_{2}+a_{3} s_{1} s_{2}}{d_{2}+b_{3} s_{1} s_{2}}\end{array}$ & $\begin{array}{l}\mathrm{MC}_{5}=\frac{n_{5}}{d_{5}}= \\
\frac{n_{4}+a_{4} s_{1}^{2}+a_{5} s_{2}^{2}}{d_{4}+b_{4} s_{1}^{2}+b_{5} s_{2}^{2}}\end{array}$ & $\begin{array}{l}\mathrm{MC}_{6}=\frac{n_{6}}{d_{6}}= \\
\frac{n_{5}+a_{6} S_{1}^{2} s_{2}+a_{\gamma_{1}} \mathrm{~s}_{2}^{2}{ }_{2}}{d_{5}+b_{6} s_{1}^{2} s_{2}+b_{7} s_{1} s_{2}^{2}}\end{array}$ & $\begin{array}{l}\mathrm{MC}_{7}=\frac{n_{7}}{d_{7}}= \\
\frac{n_{6}+a_{8} s_{1}^{3}+a_{9} s_{2}^{3}}{d_{6}+b_{8} s_{1}^{3}+b_{9} s_{2}^{3}}\end{array}$ \\
\hline$\overline{k_{1}}$ & 12.7606 & 78.2311 & 3752 & 209.906 & 93.8832 & 140.587 & 95.5636 \\
\hline$k_{2}$ & 12.7822 & 18.4840 & 795.02 & 40.8092 & 18.2299 & 26.9040 & 18.2195 \\
\hline$k_{3}$ & 13.6154 & 5.7961 & 151.64 & 7.6896 & 3.4354 & 5.1562 & 3.4774 \\
\hline$k_{4}$ & 12.3477 & 1.7286 & 28.18 & 1.5704 & 0.7014 & 1.0570 & 0.7084 \\
\hline$k_{5}$ & 7.0490 & 0.2716 & 4.46 & 0.3108 & 0.1391 & 0.2085 & 0.1411 \\
\hline$a_{0}$ & 51.6519 & -16.7617 & 0 & 0.1398 & 0.2701 & 1.0901 & 3.5421 \\
\hline$a_{1}$ & 0 & 30.4408 & 0 & 14.6919 & -2.1112 & -3.8297 & -10.9563 \\
\hline$a_{2}$ & 0 & 10.8579 & 0 & -47.1364 & -0.1734 & -0.8993 & -3.1875 \\
\hline$a_{3}$ & 0 & 0 & 0 & 21.1747 & 5.1282 & 4.0541 & 7.2717 \\
\hline$a_{4}$ & 0 & 0 & 0 & 0.9023 & 13.7577 & 11.2346 & 19.8612 \\
\hline$a_{5}$ & 0 & 0 & 0 & 0 & 2.3344 & 1.8625 & 3.0467 \\
\hline$a_{6}$ & 0 & 0 & 0 & 0 & 0.9959 & 0.9860 & 4.9172 \\
\hline$a_{7}$ & 0 & 0 & 0 & 0 & 0 & 1.2611 & 2.0797 \\
\hline$a_{8}$ & 0 & 0 & 0 & 0 & 0 & 0 & 13.2110 \\
\hline$a_{9}$ & 0 & 0 & 0 & 0 & 0 & 0 & 1.3993 \\
\hline$b_{1}$ & 0 & -1.4981 & 0 & -0.0768 & -0.5434 & -1.1783 & -0.3609 \\
\hline$b_{2}$ & 0 & 4.3819 & 0 & 0.2388 & 1.6361 & 3.8707 & 4.5648 \\
\hline$b_{3}$ & 0 & 0 & 0 & 0.0044 & -0.3751 & -0.8944 & 0.2657 \\
\hline$b_{4}$ & 0 & 0 & 0 & 0 & 0.0959 & 0.2900 & -0.1739 \\
\hline$b_{5}$ & 0 & 0 & 0 & 0 & 0.2754 & 0.0339 & 0.6215 \\
\hline$b_{6}$ & 0 & 0 & 0 & 0 & 0 & -0.0366 & -0.6978 \\
\hline$b_{7}$ & 0 & 0 & 0 & 0 & 0 & 0.1122 & 0.1626 \\
\hline$b_{8}$ & 0 & 0 & 0 & 0 & 0 & 0 & 0.1698 \\
\hline$b_{9}$ & 0 & 0 & 0 & 0 & 0 & 0 & 1.2659 \\
\hline RMS error & 2201.3 & 590 & 150.4 & 63.0 & 62.7 & 61.3 & 61.3 \\
\hline
\end{tabular}




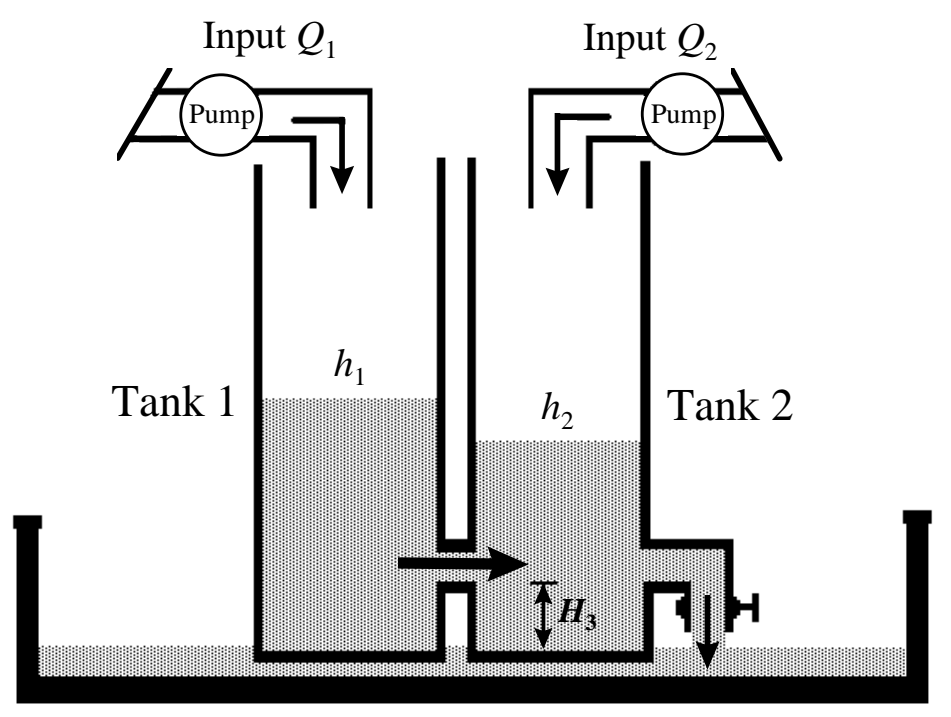

Fig. 1 A nonlinear coupled liquid-level system 

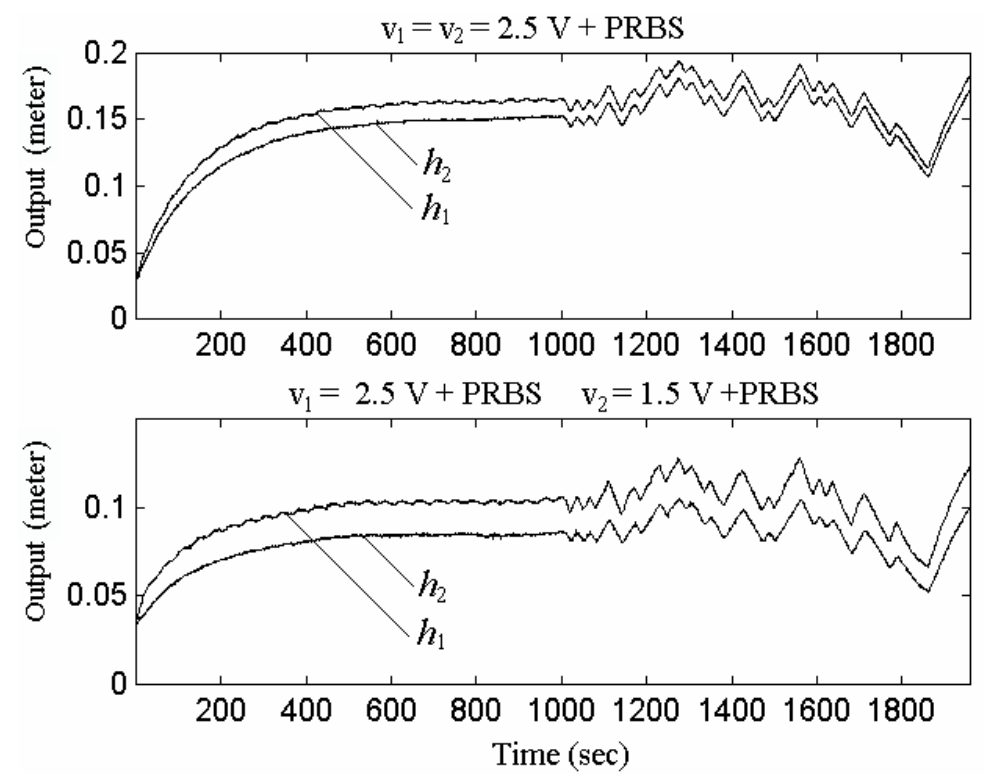

Fig. 2 Noisy responses of the physical system to steps and PRBS exciting the relevant frequencies 

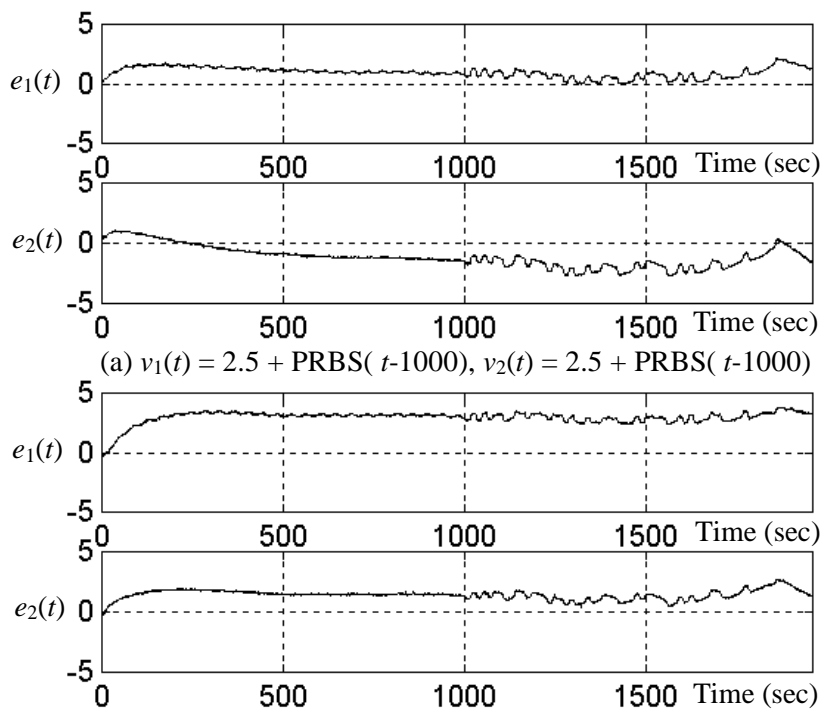

(b) $v_{1}(t)=2.5+\operatorname{PRBS}(t-1000), v_{2}(t)=1.5+\operatorname{PRBS}(t-1000)$

Fig. 3 Error residuals (in cm) of the measured clear-box model at different operating levels 

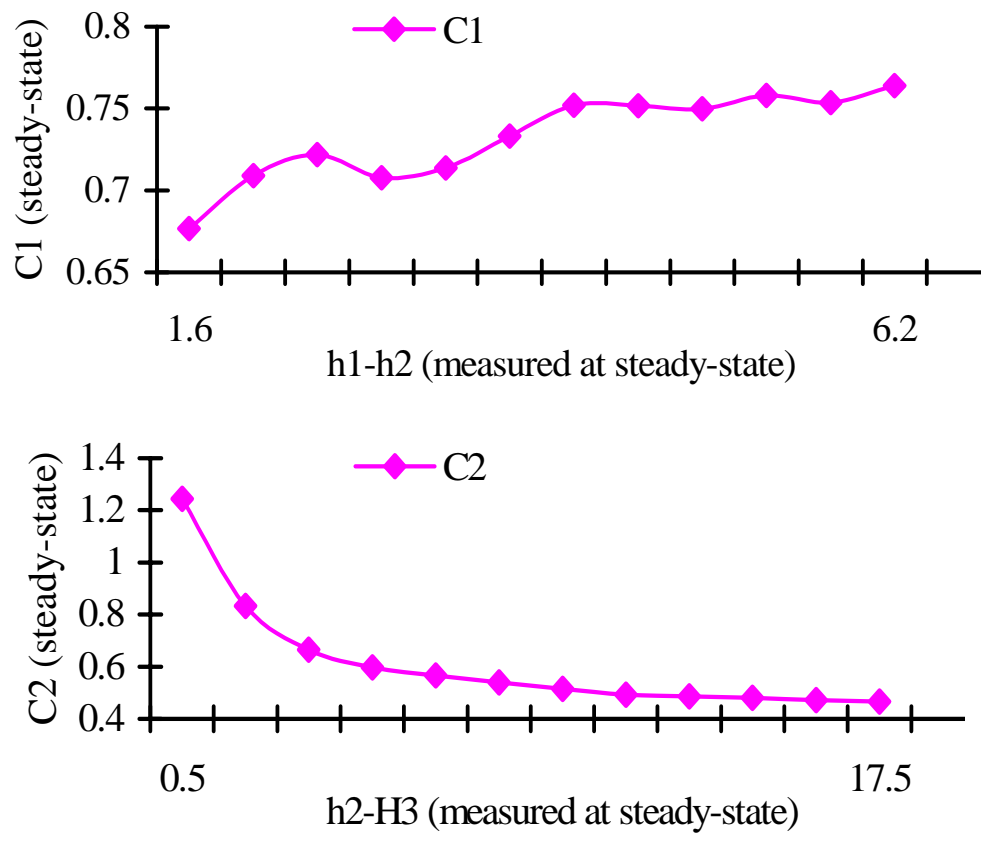

Fig. 4 Operating level dependent 'coefficients' in a nonlinear clear-box model 

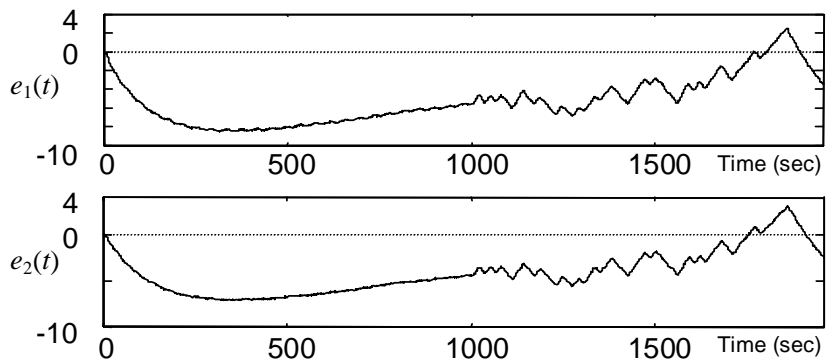

(a) $v_{1}(t)=2.5+\operatorname{PRBS}(t-1000), v_{2}(t)=2.5+\operatorname{PRBS}(t-1000)$
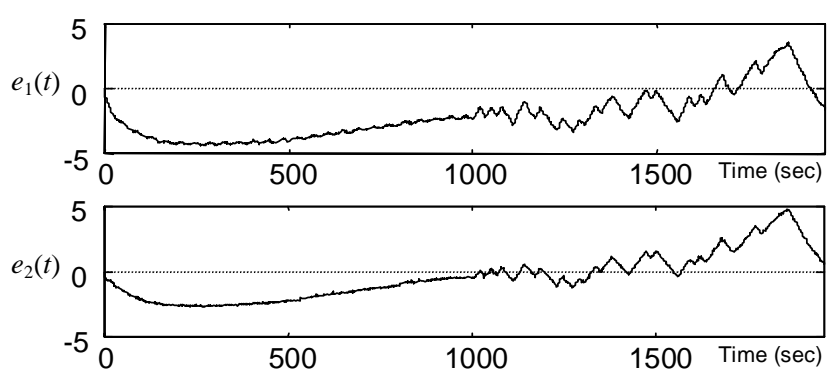

(b) $v_{1}(t)=2.5+\operatorname{PRBS}(t-1000), v_{2}(t)=1.5+\operatorname{PRBS}(t-1000)$

Fig. 5 Error residuals (in cm) of the clear-box model identified by Quasi-Newton's method 


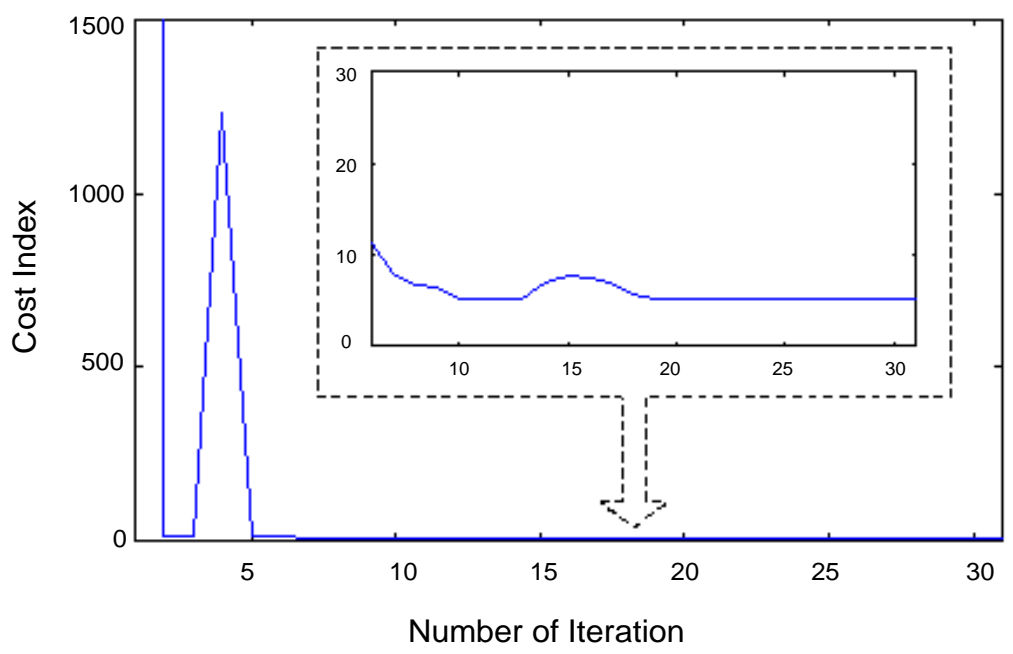

Fig. 6 Local convergence of the Quasi-Newton's method for clear-box model identification 


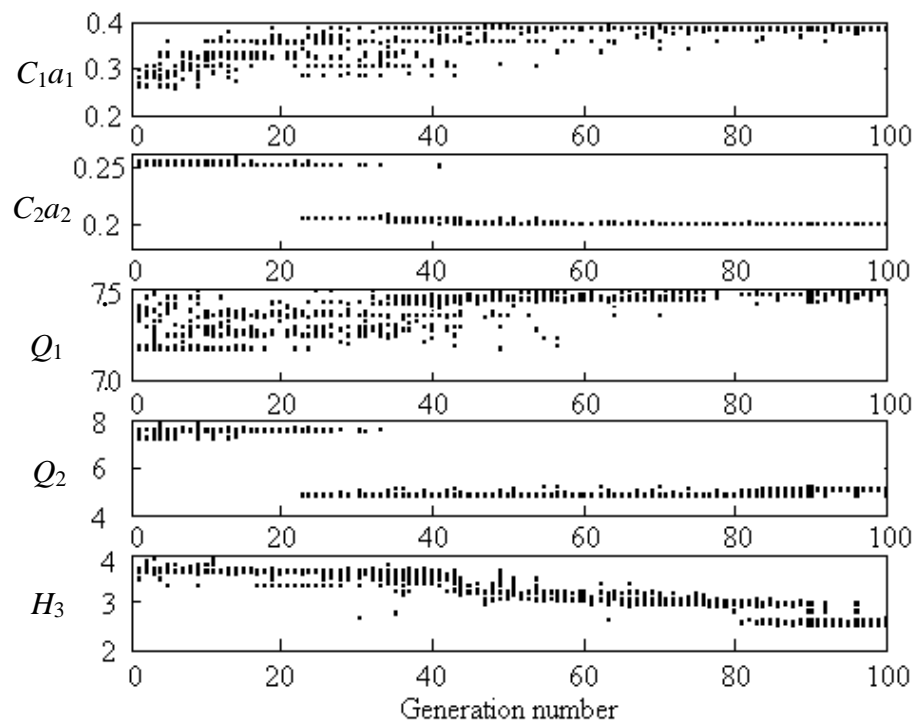

Fig. 7 Convergence trace of the best $10 \%$ parameters in each generation 

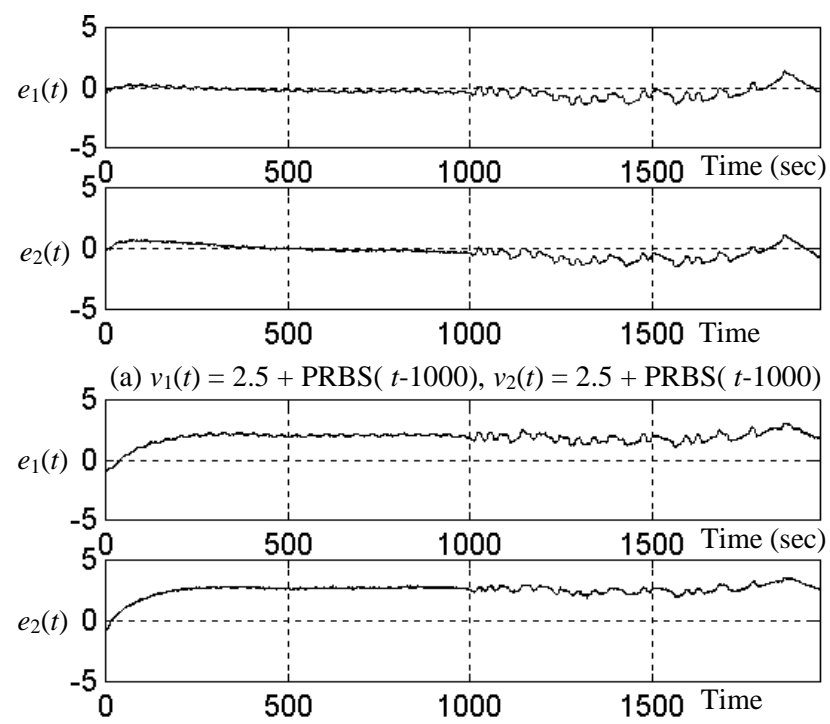

(b) $v_{1}(t)=2.5+\operatorname{PRBS}(t-1000), v_{2}(t)=1.5+\operatorname{PRBS}(t-1000)$

Fig. 8 Error residuals of the evolutionary identified clear-box model at different operating levels 

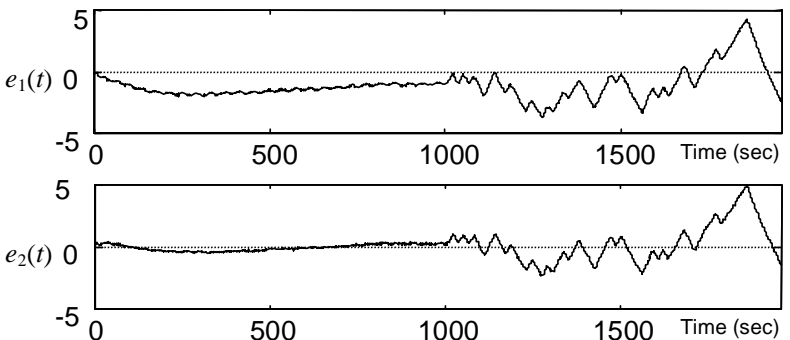

(a) $v_{1}(t)=2.5+\operatorname{PRBS}(t-1000), v_{2}(t)=2.5+\operatorname{PRBS}(t-1000)$

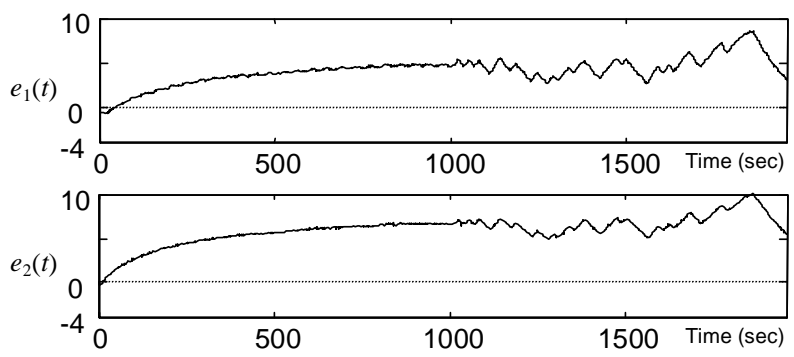

(a) $v_{1}(t)=2.5+\operatorname{PRBS}(t-1000), v_{2}(t)=1.5+\operatorname{PRBS}(t-1000)$

Fig. 9 Error residuals (in cm) of the grey-box model identified by Quasi-Newton's method 


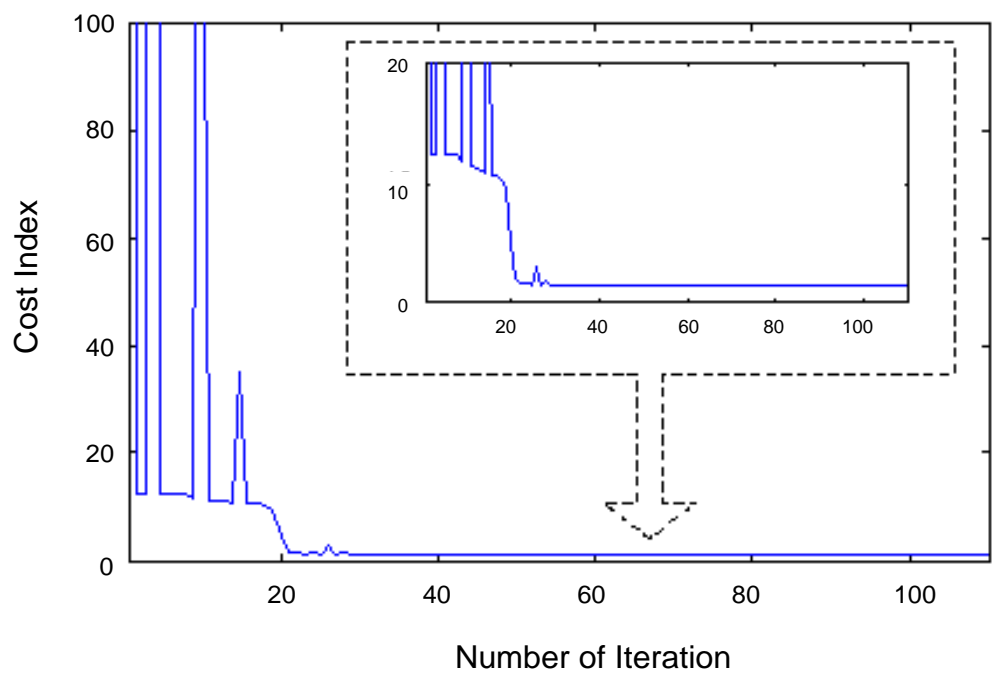

Fig. 10 Local convergence of the Quasi-Newton’s method for grey-box identification 

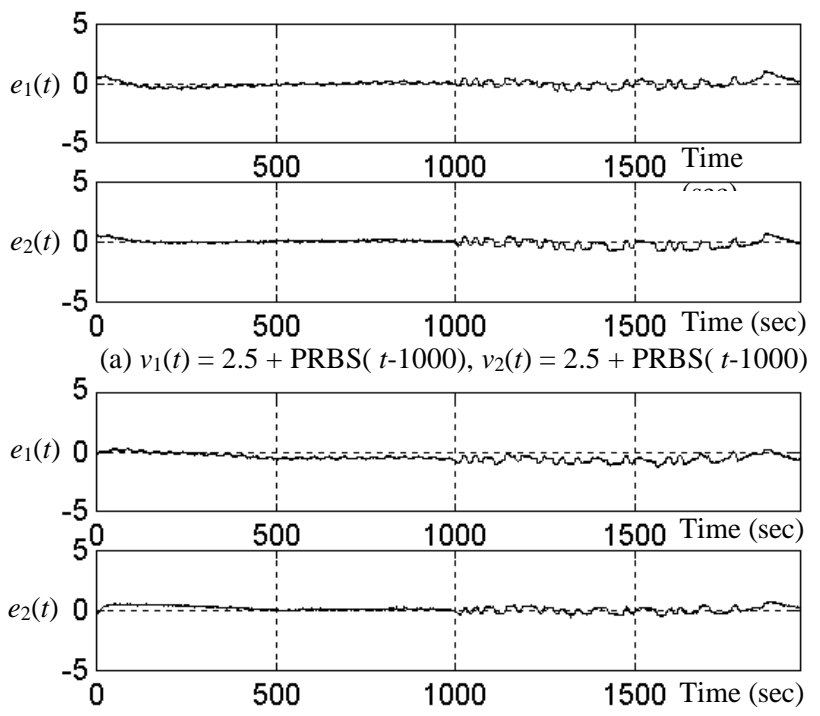

(b) $v_{1}(t)=2.5+\operatorname{PRBS}(t-1000), v_{2}(t)=1.5+\operatorname{PRBS}(t-1000)$

Fig. 11 Error residuals (in cm) of the hybrid EA identified grey-box model 

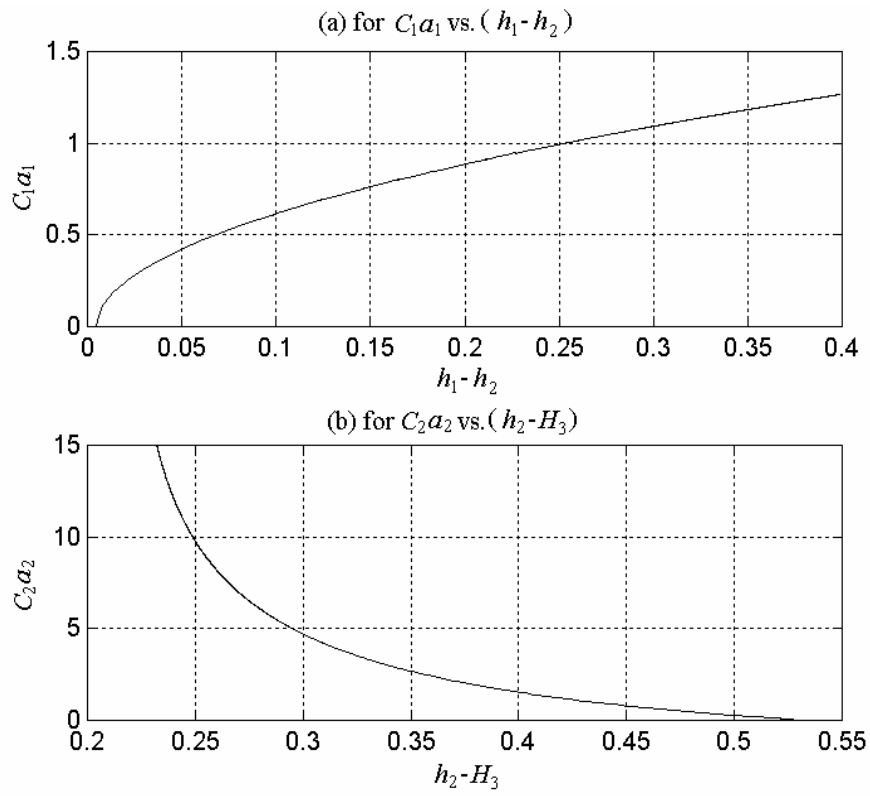

Fig. 12 Modelling the varying 'coefficients' of the clear structure in the grey-box by black-boxes 


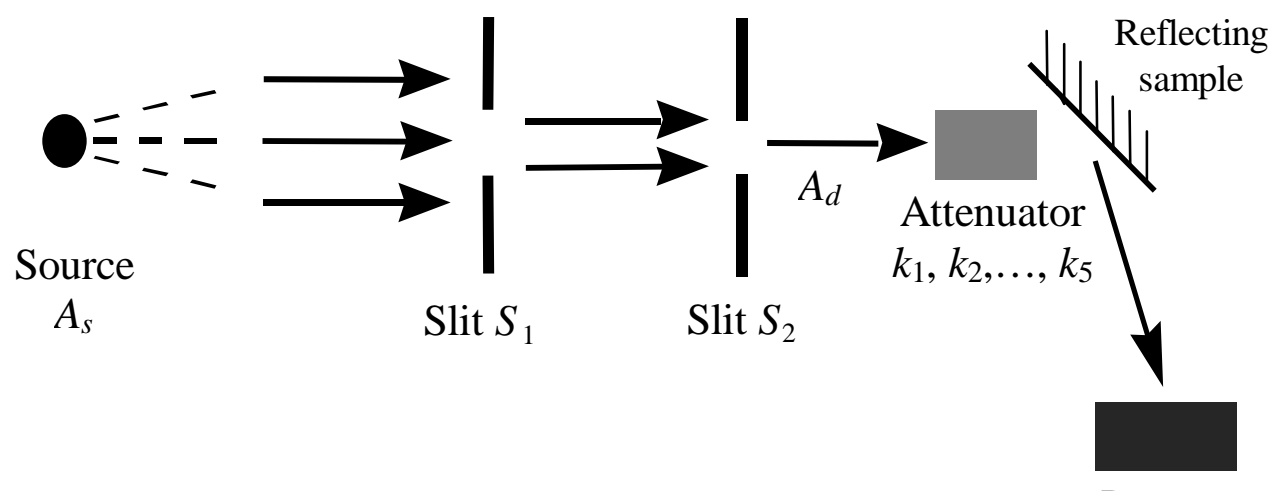

Fig. 13 A thin film reflectivity detecting system 


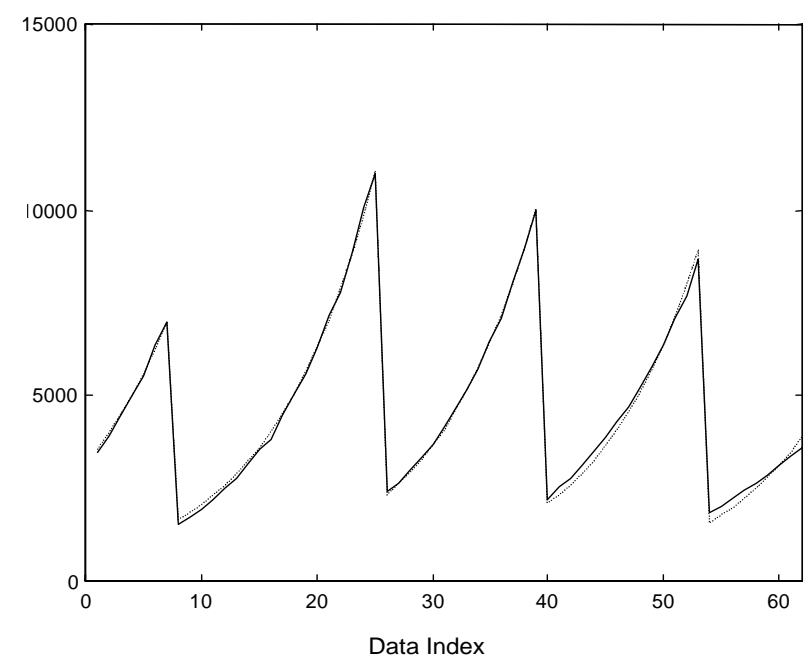

Fig. 14 Fitting acurracy of the clear-box model for data set $1 ; I\{\ldots\}$ and $\hat{I}\{-\}$ 


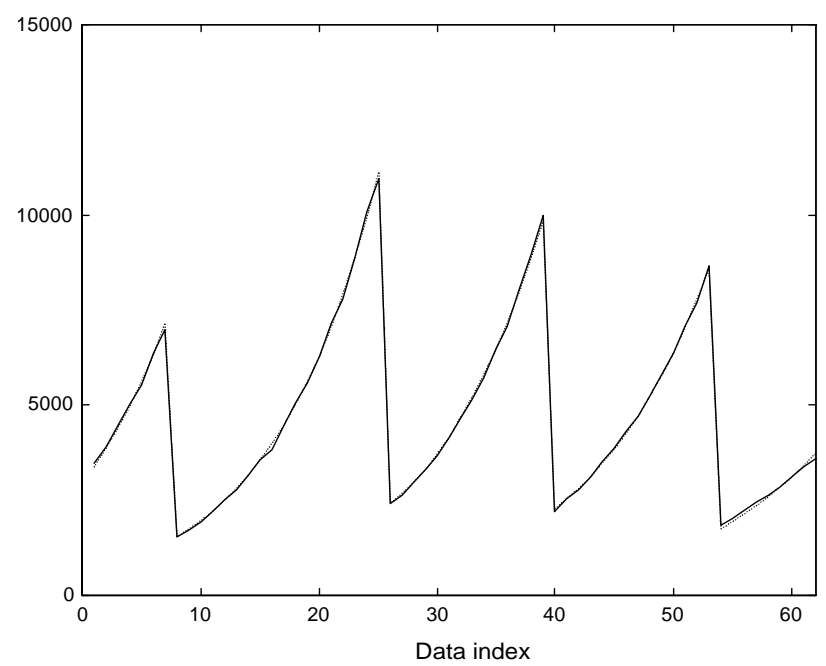

Fig. 15 Fitting acurracy of the grey-box model for data set $1 ; I\{\ldots\}$ and $\hat{I}\{-\}$ 


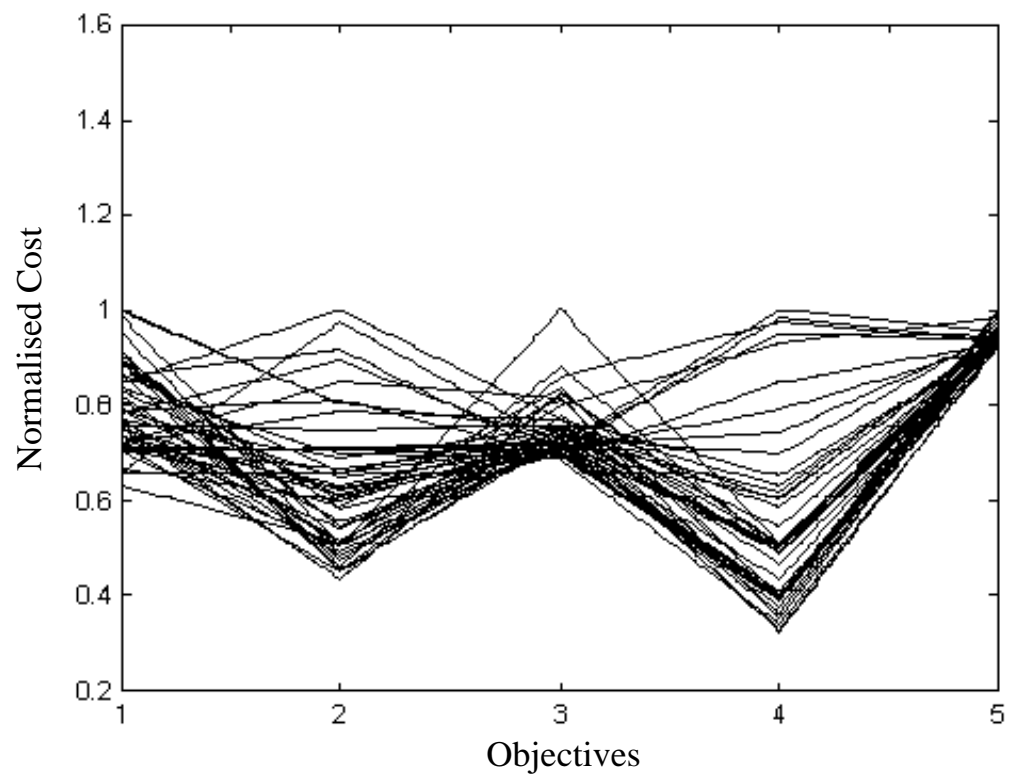

Fig. 16 Trade-offs among the different trajectory 


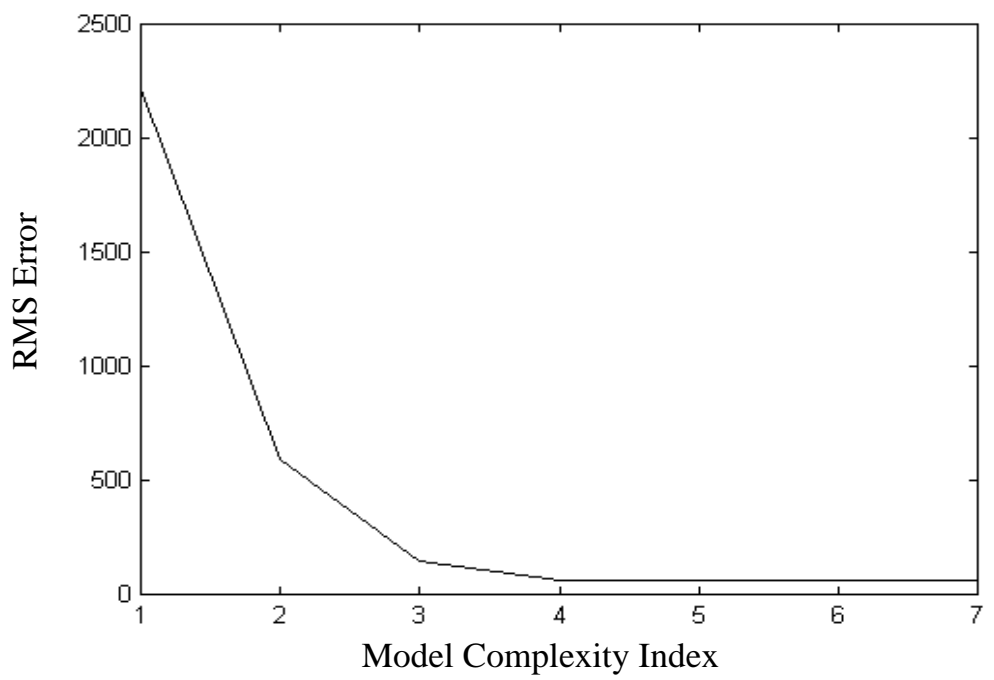

Fig. 17 Trade-offs between the model complexity index and RMS errors 